\title{
Distonía focal de mano
}

\author{
I. Proubasta ${ }^{\mathrm{I}, 2}$, J. Pagonabarraga $^{2,3}$, C. Lamas ${ }^{\mathrm{I}, 2}$, A. Millan ${ }^{\mathrm{I}}$ \\ 'Servicio de Cirugía Ortopédica y Traumatología Hospital de la Santa Cruz y San Pablo. Barcelona. España. \\ 2Profesor asociado Universidad Autónoma de Barcelona. España. \\ ${ }^{3}$ Servicio de Neurología Hospital de la Santa Cruz y San Pablo. Barcelona. España.
}

Resumen: La distonía focal de mano es un síndrome clínico neurológico caracterizado por la existencia de contracciones musculares involuntarias, sostenidas y persistentes, que provocan movimientos y posturas anormales. Su etiología, aunque controvertida, parece estar desencadenada por la actividad repetitiva, permanente y constante de la misma. Su diagnóstico es difícil, pues no existe ningún "test" o "pruebas diagnósticas" que la confirmen. De hecho, el diagnóstico de certeza de una distonía de mano puede demorarse entre 5 y 8 años, no siendo extraño que estos pacientes hayan pasado por varias cirugías previas innecesarias antes de su diagnóstico definitivo, el cual es básicamente clínico. Es por ello, que consideramos fundamental llegar a un diagnóstico precoz, con el fin evitar pérdidas innecesarias de tiempo y dinero, e iniciar el tratamiento más adecuado. En este sentido, la interacción entre cirujanos de mano, neurólogos y fisioterapeutas se hace imprescindible.

Palabras claves: Distonía Ocupacional, músico, escribiente, terapia ocupacional.

\section{Focal hand dystonia}

Abstract: Focal hand dystonia is a neurological clinical syndrome characterized by the existence of involuntary muscle contractions, sustained and persistent, causing abnormal movements and postures. Its etiology, although controversial, seems triggered by repetitive, continuous and constant activity of the hand. Its diagnosis is difficult because there is no "test" or "diagnostic tests" to confirm it. In fact, the true diagnosis of hand dystonia can take between 5 and 8 years. Sometimes these patients have undergone several previous surgeries before the diagnosis is established, which is mainly clinical. An early diagnosis, in order to avoid unnecessary loss of time and money, and starting the most appropriate treatment as soon as possible is considered fundamental when dealing with such an ilness. In this sense, the interaction between hand surgeons, neurologists and physiotherapists is essential.

Key words: Occupational dystonia, musician, clerk, occupational therapy.

Con el término genérico de distonía, se define un "síndrome clínico neurológico caracterizado por la existencia de contracciones musculares involuntarias, sostenidas y persistentes, que provocan movimientos y posturas anormales'.

No es nuestro propósito realizar una extensa revisión general sobre las distonías, pues escapa completamente del objetivo de este artículo, sino que la intención del presente trabajo es la de llamar la atención sobre un tipo en particular de distonía que afecta exclusivamente a la mano y cuya etiología, aunque controvertida, parece estar desencadenada por la actividad repetitiva, permanente y constante de la misma. La profundización en su estudio, es lo que nos ha llevado a escribir dicho artículo, pues estamos convencidos de que muchos cirujanos de mano, han tenido o tienen pacientes cuya sintomatología y clínica muy bien podrían corresponder a una distonía, cuyo diagnóstico es difícil, pues no existe ningún "test" o "pruebas diagnósticas" que la confirmen. De hecho, el diagnóstico de certeza de una distonía de mano puede demorarse entre 5 y 8 años, no siendo extraño que estos pacientes hayan pasado por varias cirugías previas innecesarias antes de su diagnóstico definitivo, el cual es básicamente clínico. Es por ello, que consideramos fundamental llegar a un diagnóstico precoz, con el fin evitar pérdidas innecesarias de tiempo y dinero, e iniciar el tratamiento más adecuado. En este sentido, la interacción entre cirujanos de mano, neurólogos y fisioterapeutas se hace imprescindible.

\section{HISTORIA}

Aunque el término "distonía" fue acuñado en 191 I por el neurólogo alemán Hermann Oppenheim, numerosas descripciones de distonía aparecieron antes en la literatura médica con diversos términos.
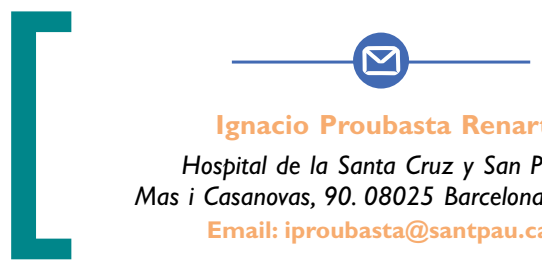

Ignacio Proubasta Renart Hospital de la Santa Cruz y San Pablo Mas i Casanovas, 90. 08025 Barcelona. España Email: iproubasta@santpau.cat 
Oppenheim creía que la distonía era provocada por una anomalía del tono muscular, en el que se producían de forma aberrante, fases de hipertonía e hipotonía ${ }^{2}$. Aunque fue Charles Bell quien describió por primera vez en 1833 la alteración en el movimiento de la mano que pueden presentar las personas que escriben continuamente y a la que llamó "calambre o parálisis de los escribientes"," no fue hasta 1836, en que Kopp describió magistralmente las características clínicas de esta distonía ${ }^{4}$. Más tarde, Gowers en 1888 , se refirió a la alteración del movimiento en pianistas y violinistas como consecuencia de una práctica excesiva, motivo por el cual se la denominó "calambre del músico"5. Al respecto, Robert Schumann escribió en su diario (1830) que después de haber tocado durante mucho tiempo el piano y con una mala postura de la mano, le falló el control del movimiento cuando quiso volver a tocarlo. Todo parece indicar que el genial músico padecía una distonía focal ${ }^{6}$. Sin embargo, otras profesiones y/u ocupaciones pueden provocarla, como las costureras, peluqueros, diseñadores gráficos, croupiers, etc., así como también en determinados deportes, como el golf o el tenis.

En el año 1908, Schwalbe emplea el término "calambres tónicos", describiendo su contribución heredita$\mathrm{ria}^{7}$. Sin embargo, bajo la influencia de Freud, la distonía era considerada predominantemente como un trastorno psiquiátrico, y ello fue así durante las primeras décadas del siglo XIX. Este hecho no resulta sorprendente si tenemos en cuenta que la distonía psicógena era relativamente común en el conjunto de las enfermedades neurológicas de la época, donde dicho síndrome fue considerado como una expresión somática de conflictos emocionales o psicológicos.

No fue hasta 1944, en que la distonía fue etiquetada por Herz como un trastorno neurológico cierto. A través de la cinematografía, el autor tipificó los movimientos distónicos como lentos, sostenidos y con fuertes contorsiones, tanto del tronco como de las extremidades ${ }^{8}$. Sin embargo, en 1984, el Comité de la Fundación de Investigación Médica de las Distonías (André Barbeau, Donald B. Calne, Stanley Fahn, C. David Marsden, John Menkes, y G. Frederick Wooten), definió la Distonía tal como se enuncia al principio de la introducción de este artículo.

\section{EPIDEMIOLOGÍA}

En EE.UU, la prevalencia general de la distonía primaria generalizada es de 3,4 por cada 100.000 indi- viduos, mientras que la de la distonía primaria focal es del 29,5 por cadal00.000. En cambio, en Europa, la prevalencia anual de todo tipo de distonías es de 15,2 por cada 100.000 individuos, siendo la distonía focal la más frecuente 9 . Al respecto, la prevalencia del calambre del músico se sitúa en el $1 \%$, cifra no despreciable si tenemos en cuenta que en el $38 \%$ de los casos no podrán volver a tocar su instrumento profesional, aun a pesar del tratamiento empleado ${ }^{10}$. En España, se estima que hay entre 25.000 y 30.000 personas afectadas de algún tipo de distonía.

La distonía, incluyendo todas sus formas, es el tercer trastorno del movimiento más común después del Parkinson y el temblor esencial.

En cuanto al género, parece ser que la distonía, en todas sus formas, incluyendo las de base genética, es más frecuente en mujeres. Sin embargo, esta relación está más cerca de I:I, cuando la distonía es focal, como la de mano". Por lo que respecta a la edad, la distonía está relacionada con la localización anatómica de la misma, siguiendo un gradiente craneocaudal. Así, la edad media de presentación del blefaroespasmo es a los 58 años, la distonía oromandibular a los 53 años, la disfonía espasmódica a los 46 años, la distonía cervical a los 45 años, las distonías mano-brazo (calambre del escritor, músico, etc) entre los 30-50 años y las distonías localizadas en las piernas suele afectar a sujetos menores de 20 años $^{12}$.

\section{ETIOLOGÍA}

Los conocimientos actuales sobre la patofisiología de las distonías es limitado, aunque el aporte de estudios neurofisiológicos y de neuroimagen, han podido poner de manifiesto una serie de alteraciones en el procesamiento de la información, procesos corticales y subcorticales de inhibición, así como también la influencia de estímulos sensitivos en la excitabilidad cortical asociados a esta patología 13,14 .

En la actualidad, existen tres líneas de investigación para explicar el sustrato fisiopatológico de la distonía, no estando claro que estén relacionadas entre sí, ni tampoco si una es más influyente que la otra ${ }^{15}$. Estas son:

\section{Pérdida de inhibición}

La pérdida de inhibición es la probable responsable de la aparición de movimientos excesivos en pacientes con distonía. Estos movimientos incluyen un aumento 
de la actividad electromiográfica, la co-contracción antagonista de los músculos y un desbordamiento (overflow) en los músculos no actuantes en la tarea específica que se realiza, es decir, al tratar de mover un dedo se provoca el movimiento de los demás e incluso de la musculatura más proximal. La pérdida de la inhibición en la distonía fue demostrada por vez primera en los reflejos espinales y del tronco medular. Ejemplo de ellos es la pérdida de la inhibición recíproca de la extremidad superior en pacientes con distonía focal de mano (DFM), entendiéndose como inhibición recíproca, la inhibición activa de los músculos antagonistas que acompañan siempre la contracción voluntaria de un músculo (agonista) ${ }^{16}$. En condiciones normales, cada vez que un músculo del cuerpo humano se contrae, simultáneamente se relaja el músculo que realiza la función opuesta, el músculo antagonista; así, al contraerse el braquial anterior, que se encarga de flexionar el antebrazo sobre el brazo, se relaja el tríceps, que realiza la función contraria, extendiéndolo. Pues bien, en los pacientes con distonía, no se produce la relajación del músculo antagonista, el que realiza la función opuesta. La contracción simultánea de dos músculos de funciones diferentes genera la alteración del movimiento que llamamos distonía ${ }^{17}$.

\section{Plasticidad cerebral anormal}

Se cree que las representaciones neuronales son moldeadas por experiencias previas a través de procesos fisiológicos, conocidos en conjunto con el nombre de "Neuroplasticidad". Numerosos estudios han puesto de manifiesto que las representaciones neuronales pueden ser alteradas en el paciente con distonía, incluyendo la DFM. Una de estas modificaciones es la anómala representación somatosensorial de los dedos en la corteza cerebral18.

Es muy posible que un incremento en la neuroplasticidad surja de la disminución de inhibición. Esta probable relación puede constituir un eslabón muy importante a la hora de demostrar que los factores ambientales pueden desencadenar la distonía. En el caso de la DFM, la actividad repetitiva durante un largo periodo de tiempo sería el factor ambiental desencadenante. Ahora bien, en qué medida la distonía está determinada por factores ambientales o condicionada genéticamente sea o no identificable, es motivo aún de gran discusión.

\section{Función sensorial anormal}

Aunque no existe una aparente pérdida sensorial a nivel clínico, algunos tests para comparar la discriminación espacial y temporal, han mostrado sutiles anor-

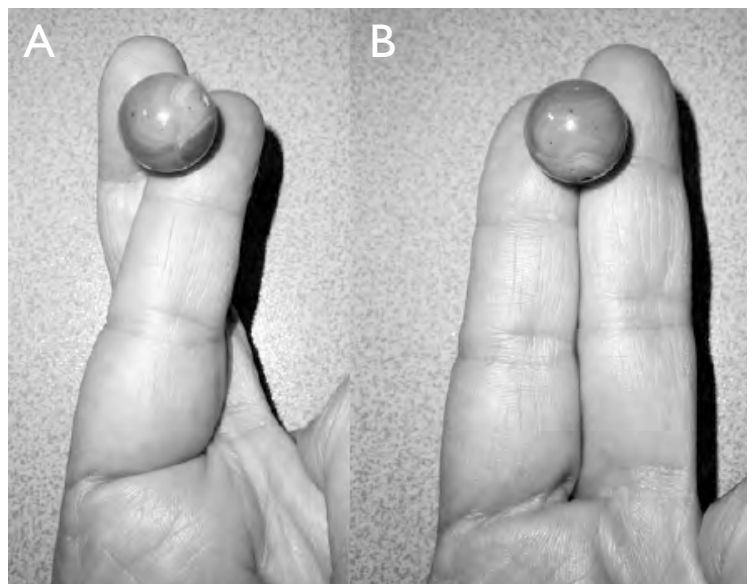

Figura I. Ilusión de Aristótoles. Cuando se cruzan los dedos A. La esfera se percibe como dos objetos, mientras que con los dedos juntos B. La esfera se percibe como uno solo.

malidades en las distonías. Éstas están presentes en ambas manos en pacientes con DFM unilateral y también en las manos de pacientes con distonía cervical y/o blefaroespasmo. Es muy posible que esta alteración sensorial esté relacionada con un anormal estado fisiológico pre-existente ${ }^{19,20}$.

En la DFM, la representación cortical somatosensorial de los dedos está disminuida. En este sentido, puede ser útil la realización de un sencillo test clínico basado en la "llusión tactil de Aristóteles". En esta ilusión, un objeto (por ejemplo una esfera) que toca el punto de contacto entre dos dedos cruzados, se percibe como dos objetos, mientras que si lo hace con los dos dedos juntos, se percibe como un solo objeto (2) Figura I. La ilusión de tener una doble sensación cuando los dedos están cruzados es debida a que el punto de contacto entre ambos corresponde a dos regiones de piel no adyacentes y funcionalmente no relacionadas, por lo que envían señales sensoriales a puntos separados de la corteza cerebral. En cambio, en los pacientes con DFM, la ilusión táctil de Aristóteles se ve marcadamente reducida en los dedos que no están afectados por el proceso, fundamentalmente entre el $4^{\circ}$ y $5^{\circ}$ dedos. Este hallazgo sugiere que en la DFM, la percepción táctil del $5^{\circ}$ dedo prevalece sobre el $4^{\circ}$ cuando los dedos se encuentran cruzados, por lo que el objeto se percibe como uno solo en vez de ser dos. Este hallazgo se correlaciona bastante bien con la severidad del déficit motor ${ }^{21}$.

A partir de la información sobre estas tres líneas de trabajo, pueden introducirse implicaciones terapéuticas importantes, tal como veremos en el apartado de tratamiento. 


\section{CLASIFICACIÓN}

Aunque la definición clínica de la distonía no ha variado desde hace años, la distonía puede clasificarse según: I) etiología (primaria o secundaria); 2) edad de presentación; 3) distribución anatómica: a) focal (calambre del escribiente y/o del músico, blefaroespasmo, cervical, etc.), b) segmentaria, con el compromiso de 2 o más regiones contiguas, c) multifocal, d) hemidistonía, con compromiso de un hemicuerpo, y e) generalizada); y 4) según su curso clínico $22,23$.

Sin embargo, estudios más recientes, han redefinido estos sistemas de clasificación básica, impulsados en gran medida por la evolución que ha experimentado la genética. En cierto sentido, la genética ha sesgado el estudio de las distonías. Así, las distonías generalizadas suelen afectar a niños, en los que un subgrupo de ellos se deben a la mutación $\triangle \mathrm{GAG}$ en el exón 5 del gen TORIA (DYTI). EITORIA fue el primer gen que se asoció casualmente con una distonía primaria. Sin embargo, se han observado otras formas genéticas de distonía como DYT5, DYTI I y DYT I2, que presentan como denominador común, el hecho de responder favorablemente al tratamiento, especialmente con la estimulación cerebral profunda (2) Tabla I. Es interesante hacer constar que algunos músicos con distonía focal, entre el $10 \%$ y el $25 \%$, tienen antecedentes familiares con historia clínica de distonía, por lo que puede considerarse un factor genético en algunos pacientes.

En el presente artículo, nos centraremos única y exclusivamente en la DFM, siendo los calambres del escribiente y/o del músico las más frecuentes, aunque también aparecen en otras profesiones, deportes o determinados trabajos susceptibles a provocarlas. De ahí, que a este tipo de distonías se las conozca actualmente con el término general de "Distonías Ocupacionales"16.

\section{DIAGNÓSTICO}

\section{Examen clínico}

La valoración de una probable DFM, se inicia con una completa historia clínica, incluyendo una detallada y exhaustiva búsqueda de todos los antecedentes familiares y personales, especialmente la toma de fármacos y/o drogas. Asimismo, la profesión del paciente, puede proporcionar una información esencial sobre la patología que presenta. Así, profesiones como músicos, administradores que teclean durante muchas horas el ordenador, tareas que requieran de la repetición de

\section{TABLA I - CLASIFICACIÓN DE LAS DISTONíAs}

I. POR SU ETIOLOGÍA:

\section{I.I. Primaria:}

- Distonía por torsión - PTD - DYTI - TORYIA

- Cervical/craneal - DYTI3

- Disfonía - DYT4

- Tortícolis familiar del adulto - DYT7

- Mixta - distonía fenotípica - DYT6

- Esporádica, distonía focal: Blefaroespasmo.

Oromandibular.

Espasmódica.

Cervical.

Ocupacional.

Del tronco.

Otras.

I.2. Síndromes de distonía plus:

- Distonía asociada al Parkinson.

- Distonía con mioclonus.

\subsection{Secundaria:}

- Lesión perinatal cerebral.

- Malformación congénita.

- Infecciosa, postinfecciosa, inflamatoria o paraneoplásica.

- Traumatismo craneal, cirugía cerebral.

- Talamotomía.

- Lesión cervical.

- Estenosis del canal lumbar.

- Lesión eléctrica.

- Lesión nerviosa periférica.

- Tumores de la fosa posterior.

- Malformación cerebral arteriovenosa.

- Tumor cerebral.

- Esclerosis múltiple.

- Inducida por fármacos y/o drogas.

- Metabólica

- Otras.

I.4. Heredodegenerativa.

I.5. Psicógena.

2. POR SU EDAD DE PRESENTACIÓN:

2.I. Infantil: de inicio hasta los 12 años de edad.

2.2. Juvenil: entre los 13 y 20 años. A este grupo pertenecen mayoritariamente las distonías secundarias, especialmente la parálisis cerebral infantil y las enfermedades degenerativas metabólicas.

2.3. De inicio en adultos: a partir de los 20 años de edad. Estas distonías suelen ser de localización focal y de curso clínico estático, esto es, que no tienen tendencia a propagarse. En el caso de las distonías ocupacionales, la incapacidad funcional está relacionada con la localización de los espasmos musculares distónicos, pudiendo interferir con la actividad laboral de los enfermos, como en el caso del calambre del escribiente, del músico, etc.

3. POR SU DISTRIBUCIÓN ANATÓMICA:

3.I. Focal:

- Blefaroespasmo.

- Oromandibular.

- Cervical.

- Laríngea.

- Mano: calambre del escribiente, del músico, ocupacional, etc.

\subsection{Segmentaria:}

- Craneal: se afectan dos o más partes del cuerpo contiguas.

- Axial: se afecta el cuello y tronco.

- Braquial: se afecta un brazo; ambos brazos, +/- cuello, +/-tronco.

- Crural: se afecta una pierna y tronco; ambas piernas, + - tronco. 


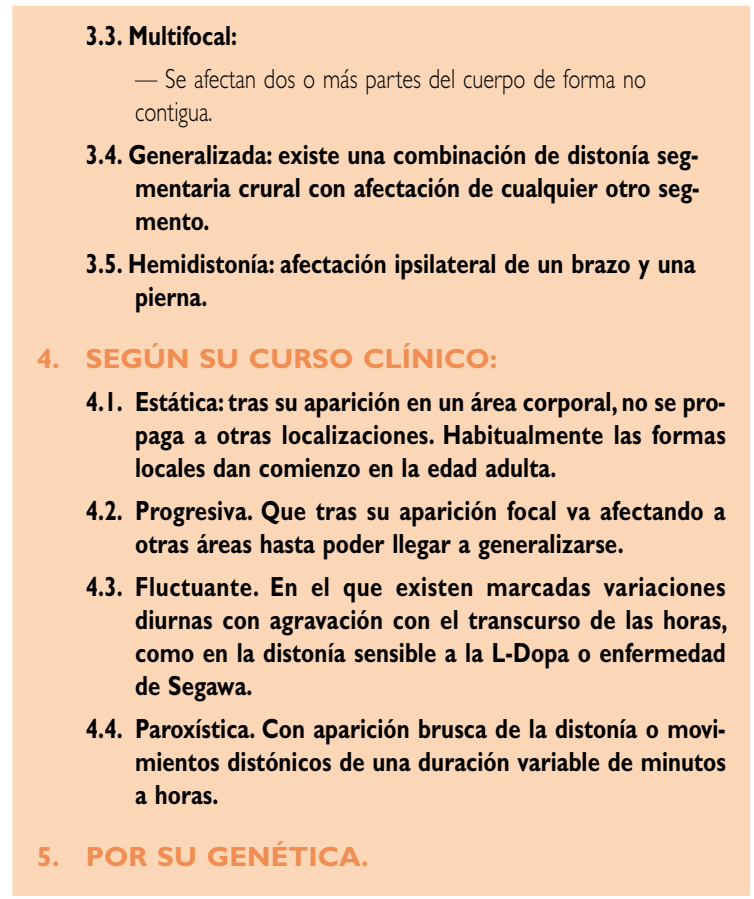

gestos y esfuerzos en la mano, como es el de estar en una cadena de montaje, indican a menudo el origen del problema. En este contexto, muchos casos de distonías focales se inician de forma gradual, mientras que las distonías secundarias suelen expresarse de forma aguda o subaguda ${ }^{24,25}$.

En la DFM, no existe una patrón uniforme sintomático, pues el espectro clínico es muy variable. En general, se trata de una persona, sea hombre o mujer, entre los 30 y 50 años de edad, aunque no es infrecuente que ocurra en pacientes más jóvenes, que acuden a la consulta con una clínica un tanto abigarrada. El síntoma principal es la aparición de una contracción muscular involuntaria de la mano, tras realizar un gesto, esfuerzo o maniobra repetitiva concreta durante un cierto periodo de tiempo ${ }^{26}$. La DFM no se asocia con dolor, si bien se han descrito mialgias posteriores al espasmo muscular prolongado. En el caso del calambre del músico, el paciente describe perfectamente lo que le pasa. Al respecto, existe una incoordinación motora entre el dedo o los dedos que ejecutan la acción de tocar, ya sea el piano, gitarra, violín, etc., con el resto (- Figura 2. En el calambre del escribiente, el síntoma inicial suele ser una sensación de torpeza durante la escritura, con disminución de la velocidad y de la fluencia del movimiento. Asimismo existe una tendencia a asir el lápiz de forma muy apretada, lo que comporta la rápida fatiga de la mano y la aparición de una contractura de la misma e incluso a nivel

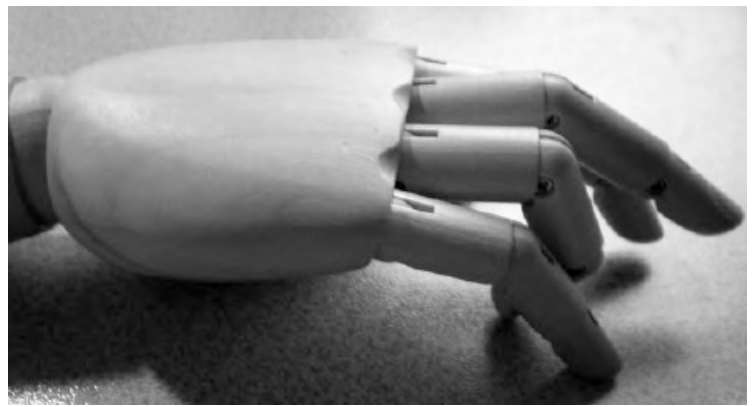

Figura 2. Simulación de la postura de los dedos de una mano en una distonía focal del músico tocando el piano. Obsérvese que el cuarto dedo se halla en flexión, en clara distonía, mientras el medio presenta una extensión compensatoria.

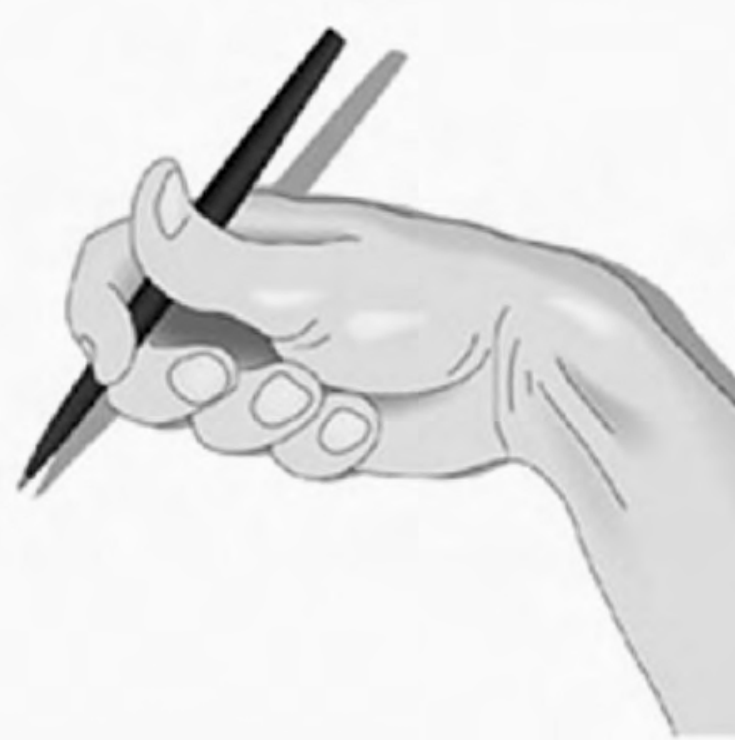

Figura 3. Representación pictórica de la postura anómala de dedos y muñeca en un caso de DFM.

más proximal @ Figura 3. Ello hace que aparezca una anómala postura de muñeca y/o dedos, los cuales pueden flexionarse o extenderse excesivamente. En los demás casos de DFM ocupacional, la forma de presentación puede ser muy variada, desde una debilidad de la mano, enlentecimiento o tremor digital, e incluso dolor inespecífico ${ }^{27}$.

Dicha sintomatología suele ser gradual y afectar a una sola mano, aunque pueden involucrar las dos. Al principio, la clínica desaparece al cabo de pocos segundos después de suspender la actividad que se realiza. Sin embargo, en el curso de los meses, las contracciones y espasmos musculares se hacen más frecuentes, esto es, al poco rato de iniciada la actividad, requiriendo más tiempo de reposo para volver 
de nuevo a realizar la misma. Dicha evolución es variable, si bien progresa durante los 6-12 primeros meses, para luego estabilizarse. Sin embargo, se han descrito casos que empeoran años después. Es común que estos pacientes hayan sido previamente intervenidos quirúrgicamente, incluso varias veces para solucionar su problema. Así, no es extraño observar incisiones en la palma de la mano y/o muñeca, con motivo de supuestos síndromes del túnel carpiano, tendinitis de DeQuervain, síndrome de intersección, síndrome de Wartenberg (neuritis de la rama sensitiva del nervio radial), etc., o a nivel del codo por epicondilitis, síndrome del pronador redondo o para descomprimir el nervio radial en la arcada de Frohse, lo que todo ello dificulta aún más el diagnóstico. En general, estas cirugías suelen ser ineficaces, pues los pacientes continúan teniendo los mismos problemas previos.

Es importante resaltar que, ante la ausencia de una postura anómala, aun cuando exista contractura o espasmo muscular, no puede considerarse una distonía. En este sentido, otra patologías musculares pueden estar presentes, como son los casos de síndrome compartimental crónico o por esfuerzos repetitivos (sobreuso), los cuales cursan con dolor y contractura muscular, pero no con postura anómala de la mano ${ }^{28}$.

Uno de los aspectos a tener en cuenta en la DFM, es que el movimiento distónico tiende a desaparecer si la parte del cuerpo afectada, en nuestro caso la mano, se coloca en posición de reposo. Este hallazgo es de suma importancia en el diagnóstico diferencial, pues los movimientos involuntarios del clonus miopático, la corea o el Parkinson, no desaparecen cuando la mano se halla en posición neutra de reposo.

Analizando las posturas anómalas, es obvio que las mismas son consecuencia de un problema del control motor, especialmente en el control individual de los dedos. Así, el hecho de querer mover repetidamente un dedo en concreto, desencadena el movimiento involuntario de los otros e incluso en la musculatura más proximal, ya sea en la muñeca y/o antebrazo, dando lugar a estas posturas anómalas, signo que identifica a la distonía. Otro de los hallazgos adicionales de la DFM, observable comúnmente en la distonía cervical, es la provocación del gesto antagonista a partir de un truco o engaño sensorial, cual es la de tocarse suavemente una parte de la mano, aunque puede ser en otro sitio de la extremidad superior. Con dicho gesto propioceptivo, se mejora la distonía, pero solamente por un breve periodo de tiempo. Se ha descrito en el calambre del músico, que algunos pacientes se colocan un esparadrapo en el dorso de la mano como truco sensorial.

En cuanto al examen físico, es imprescindible identificar signos y/o síntomas neurológicos que sugieran otro tipo de etiología diferente a la distonía focal, como son los síndromes extrapiramidales. Dentro de éstos, existen principalmente dos grupos, uno con rigidez y trastornos posturales como en la enfermedad de Parkinson y síndromes parkinsonianos, y un segundo grupo que comprende diversos movimientos involuntarios como son la corea, atetosis, balismos, temblor, diskinesias, mioclonía o tics.

El examen de la movilidad articular, tanto de muñeca y dedos, nos pondrá de manifisto si existe una restricción del movimiento, en cuyo caso deberemos descartar la presencia de patología articular, especialmente procesos degenerativos y/o artríticos. En el lado opuesto, una hiperlaxitud articular también puede dar lugar a que la contracción muscular se convierta en el estabilizador primario de la articulación afectada por esta laxitud. En este ajuste, la necesidad prolongada de una estabilización dinámica comporta la aparición de dolor, fatiga y contractura. Además, la hipermovilidad puede conducir a la compresión de un nervio digital, neuropatía por tracción y/o a la aparición de una sinovitis traumática.

Cualquier alteración de la sensibilidad de los dedos, especialmente la hipo o hiperestesia, pueden indicar que estamos ante la presencia de un síndrome por compresión nerviosa, ya sea del nervio mediano a nivel del túnel carpiano o del nervio cubital en el canal de Guyon, aunque en casos de DFM no es infrecuente que el paciente relate sensación de hormigueo en los dedos. En estos casos, se hace necesario la práctica de una electromiografía para descartar dichas compresiones. Sin embargo, esta exploración tiene una utilidad limitada, básicamente por su inherente dificultad en coordinar la misma durante el periodo sintomático. A pesar de ello, puede resultar un instrumento de gran valor diagnóstico en la distonía focal, pues se ha observado que existe una hiperactividad de los músculos que no participan activamente en la tarea que provoca la misma.

La disminución y/o ausencia de los pulsos arteriales distales, radial y/o cubital, puede ser indicativo de diversas patologías que se acompañan de entumecimiento y calambres, como es el síndrome del martilleo hipotenar, resultado de la trombosis de la arteria cubital y/o del arco superficial palmar. 
Tabla $\|$ - Escala de Tubiana, para la evaluaCIÓN FUNCIONAL DEL CALAMBRE DEL MÚSICO

Etapa 0: Incapaz de tocar.

Etapa I: Toca notas, pero sin fluidez, con bloqueos frecuentes y dificultad.

Etapa 2: Toca secuencias de notas con lentitud y dedos "vacilantes".

Etapa 3: Toca piezas fáciles, sin grandes requerimientos técnicos.

Etapa 4: Toca casi normalmente, pero los pasajes difíciles, son evitados por miedo o aparición de síntomas motores.

Etapa 5: Ejecución normal.

Asimismo, es muy importante diferenciar la DFM de los movimientos anormales psicógenos. Aunque éstos no superan el $4 \%$ del total de los casos, se debe sospechar el origen psicógeno de una distonía cuando el comienzo es brusco, aparece en reposo desde el inicio, no empeora con la acción, no mejora con "trucos sensitivos" y su patrón es inconsistente o desaparece con la distracción. Normalmente se asocia con otras alteraciones del movimiento incongruentes y se observan hallazgos atípicos en la exploración ${ }^{29}$.

Finalmente, resulta útil poder medir la gravedad de la distonía así como evaluar los resultados obtenidos con los distintos tratamientos. Para ello, existen distintas escalas de valoración ${ }^{30}$. Sin embargo, por lo que se refiere a la DFM, las pocas existentes hacen referencia al calambre del músico. Al respecto, la más empleada es la desarrollada por Tubiana ${ }^{31,32}$, quien las estratifica en seis etapas $(0=$ incapaz de tocar $5=$ ejecución normal) (-) Tabla II.

\section{ESTUDIOS COMPLEMENTARIOS}

El examen radiográfico, debe ser la primera exploración a efectuar. Basta con proyecciones anteroposterior y lateral de muñeca y anteroposterior y oblicua de mano, para descartar inicialmente patología osteoarticular, aunque procesos degenerativos iniciales, especialmente a nivel de las articulaciones interfalángicas proximales y distales, trapeciometacarpiana, etc., pueden muy bien no detectarse radiográficamente, motivo por el cual resulta útil practicar una gammagrafía ósea, pues aunque tenga poca especificidad presenta una gran sensibilidad en detectar cualquier foco inflamatorio a nivel óseo $u$ articular.
En el caso de que estas exploraciones sean negativas, pueden solicitarse otras exploraciones, como la RMN - la ecografía, donde es frecuente observar lesiones de partes blandas, como son los gangliones escafolunares y/o la ruptura crónica del fibrocartílago triangular. Sin embargo, hay que tener mucho cuidado en interpretarlas, pues en la mayoría de ocasiones no tienen nada que ver con la sintomatología que presenta el paciente.

Tal como se ha comentado, la electromiografía puede identificar los músculos activados de forma anómala, apareciendo una pérdida del patrón alternativo normal entre agonistas y antagonistas, con cocontracción de ambos prolongada y disminución de la inhibición recíproca. Asimismo, el fenómeno de "overflow" está presente en músculos que no deberían estar implicados. Sin embargo, para que estos hallazgos estén presentes, la electromiografía debe coordinarse durante el periodo sintomático, hecho que dificulta el examen y su interpretación.

Por último, y como estudios diagnósticos complementarios, se encuentran los potenciales evocados motores y la Resonancia Magnética funcional (RMf), esto es, la observación en imágenes de las regiones cerebrales que ejecutan una acción determinada. Con los primeros se ha objetivado que el córtex motor inhibidor está alterado ${ }^{33}$, mientras que con la segunda se han podido comprobar anomalías en la corteza cerebral, los ganglios basales y cerebelo, hipotetizando que en las distonías focales, como la DFM, existe una desconexión entre ellas, aunque hoy por hoy no existe una etiología clara sobre su patofisiología ${ }^{34,35}$.

\section{TRATAMIENTO}

El tratamiento de la distonía depende de si ésta es primaria o secundaria. En este último caso, el tratamiento va a depender de la causa que la origina.

En la distonía primaria, como es la DFM, diversas estrategias terapéuticas han sido utilizadas, entre las que cabe destacar: I) Rehabilitación; 2) Tratamiento médico; 3) Toxina botulínica, y 4) Tratamiento quirúrgico.

\section{Rehabilitación}

La reeducación funcional, el asesoramiento genético y el tratamiento de otros trastornos afines acompañantes, incluyendo la depresión, son partes muy importantes en el enfoque terapéutico integral de estos 
pacientes ${ }^{36}$. Al respecto, la rehabilitación y las ortesis bien diseñadas, pueden mejorar la postura y prevenir contracturas.

La reeducación, permite recuperar la coordinación. En el caso de las distonías focales, como el calambre del músico, del escribiente y/u ocupacional, debe aprenderse a coordinar su acción utilizando nuevos procedimientos. La reeducación no consiste en aprender algo nuevo, sino en conseguir estabilizar un estado físico y mental que permita que el cuerpo haga lo que ya sabe hacer. Teniendo en cuenta que en la DFM existe un déficit de inhibición y una neuroplasticidad y función sensorial anómalas, se han propuesto todo una serie de actividades encaminadas a recuperar la normalidad de estas disfunciones. En este sentido, el método llamado "Retorno Sensoriomotor" ha mostrado buenos resultados en los músicos que tocan el piano. Se trata de entrenar de forma individual los dedos no afectados, mientras que los demás dedos se inmovilizan mediante férulas. Sin embargo, este método no es efectivo en pacientes que tocan instrumentos de viento ${ }^{37}$.

De forma similar, dicho método y otros, se han utilizado en el calambre del escritor, con resultados variables $^{38,39}$.

Si la distonía es consecuencia de una disfunción sensorial, todo aquello que pueda normalizar la misma puede ser beneficioso, pues secundariamente proporciona una mejor función motora. En este sentido, entrenar cada dedo con el sistema de escritura Braille puede resultar muy útilit.

Otra alternativa terapéutica, es el bloqueo anestésico muscular, pues con ello se pretende minimizar las descargas de las fibras aferentes. Una inyección de 5-10 cc de lidocaína en el músculo afectado por la distonía, puede mejorarla por un periodo de $24 \mathrm{~h}$, aunque si se simultanea con etanol, el efecto puede prolongarse varias semanas ${ }^{41,42}$

En cuanto a las ortesis, aunque mal aceptadas, especialmente en niños, en algunos casos pueden ser utilizadas como sustitutos de los denominados trucos sensoriales, de forma similar como lo haría el propio paciente al tocarse su propia mano para evitar la postura anómala ${ }^{43}$.

\section{Tratamiento médico}

El tratamiento farmacológico incluye el uso de anticolinérgicos y miorelajantes.
Los anticolinérgicos, como el Trihexifenidilo (Artane ${ }^{\circledR}$, Pacitane ${ }^{\circledR}$ ), pueden ser útiles en el tratamiento de las distonías, sean éstas generalizadas o localizadas. Cuando dicho fármaco se instaura a dosis bajas y se incrementa lentamente, suele ser bien tolerado. En general, se recomienda iniciar el tratamiento con un I mg por la noche e ir aumentando la dosis hasta los $12 \mathrm{mg}$ por día durante 4 semanas. Sin embargo, algunos pacientes necesitan hasta 60-100 mg por día, aunque pueden experimentar efectos secundarios como adormecimiento, confusión, falta de memoria y/o alucinaciones. Otro anticolinérgico comúnmente utilizado en las distonías es la Piridostigmina (Times$\operatorname{pan}^{\circledR}$, Mestinon $\left.{ }^{\circledR}\right)^{44}$.

Algunos pacientes requieren de una combinación de fármacos, como son los miorelajantes, tipo benzodiacepinas (diacepam, lorazepam o clonazepam), pues proporcionan un alivio adicional en aquellos pacientes que no responden apropiadamente a los anticolinérgicos. Sin embargo, los resultados obtenidos con dichos medicamentos son bastante aleatorios ${ }^{45}$.

\section{Toxina botulínica}

La introducción de la toxina botulínica (TB) en la década de 1980 revolucionó el tratamiento de las distonías. La TB, se ha convertido en una potente herramienta terapéutica en el tratamiento de una variedad de trastornos neurológicos y otros trastornos que se acompañan de anómalas, excesivas e inapropiadas contracciones musculares. La TB actúa produciendo un bloqueo, temporal y reversible de las terminaciones nerviosas colinérgicas, creando un estado de debilidad en el músculo inyectado. En diciembre de 1989, la Food and Drug Administration (FDA) de los EE.UU, aprobó la TB como agente terapéutico en pacientes con estrabismo, blefaroespasmo y otros trastornos del nervio facial, incluyendo el espasmo hemifacial. En diciembre de 2000, se incluyó también el Botox y la toxina botulínica B (Myobloc) en el tratamiento de la distonía cervical y, posteriormente en las distonías ocupacionales, incluyendo los calambres del escribiente y/o músico ${ }^{42}$. Aunque su aplicación más amplia se encuentra todavía en el tratamiento de los trastornos que se manifiestan por la aparición de contracciones musculares anormales, excesivas y/o inapropiadas, el uso de la TB se está expandiendo rápidamente para el tratamiento de otros procesos, incluyendo los oftalmológicos, gastrointestinales, urológicos, ortopédicos, dermatológicos y/o cosméticos ${ }^{46}$.

Cuando se utiliza TB, es importante tener en cuenta la presencia de otros trastornos neurológicos que 
podrían agravar el problema. Así, la existencia de paresias preexistentes, como en la esclerosis lateral amiotrófica, las miopatías o polineuropatías, así como en pacientes con trastornos de transmisión neuromuscular, tales como la miastenia gravis o el síndrome de Lambert-Eaton, contraindican su administración. Dejando aparte estas patologías, se ha podido demostrar que el uso prolongado de TB no produce reacciones adversas, situación que es importante conocer debido a que muchas de estas distonías son crónicas y que pueden requerir tratamientos prolongados. En cuanto a su dosis, varía dependiendo del tipo de distonía. Así, para la DFM, basta la aplicación de 5 U por cada músculo involucrado, con intervalos de 2 semanas a 4 meses. Sin embargo, si a las dos semanas no hay respuesta eficaz, puede aumentarse la dosis hasta las 40 - $50 \mathrm{U}$ /músculo.

Los resultados obtenidos con TB en los pacientes con DFM son controvertidos, pues se considera que raramente satisfacen las expectativas, especialmente en los músicos profesionales, pues aun cuando se consiga una mejoría, ésta suele ser parcial y transitoria. Por otra parte, la debilidad muscular que provoca en el músculo inyectado, hace que no se tenga la fuerza necesaria para tocar el instrumento en cuestión, lo que desmotiva al paciente a seguir intentando la terapia con $\mathrm{TB}^{47-51}$. A pesar de ello, la TB puede constituir la única alternativa eficaz cuando el tratamiento farmacológico no mejora la sintomatología.

\section{Tratamiento quirúrgico}

Existen varias opciones quirúrgicas en el tratamiento de las distonías focales, entre las que cabe destacar la estimulación cerebral profunda, especialmente del globo pálido interno, mediante procedimientos esterotácticos. Los resultados en la distonía cervical son esperanzadores, aunque dicho tratamiento, en el caso de la DFM, suele reservarse cuando los demás tratamientos resultan ineficaces ${ }^{52,53}$. Otros tratamientos quirúrgicos, como son la denervación periférica y las miectomías, se han utilizado básicamente para la distonía cervical y el blefaroespasmo ${ }^{5,55}$.

El resumen del tratamiento a seguir delante de una DFM, es que debe iniciarse el mismo mediante rehabilitación, entrando en un programa de reeducación, el cual debe intentar "purgar" la presunta mal adaptada secuencia sensoriomotora con la esperanza de desarrollar un patrón más normal. En ocasiones resulta útil suplementarla con anticolinérgicos y/o la inyección de TB. Si ello fracasa, puede emplearse la estimulación cerebral profunda, tratamiento muy prometedor pero que es preciso analizar sus resultados a largo plazo (4) Figura 4.

\section{CONFLICTOS DE INTERESES}

Los autores declaran no tener conflictos de intereses.

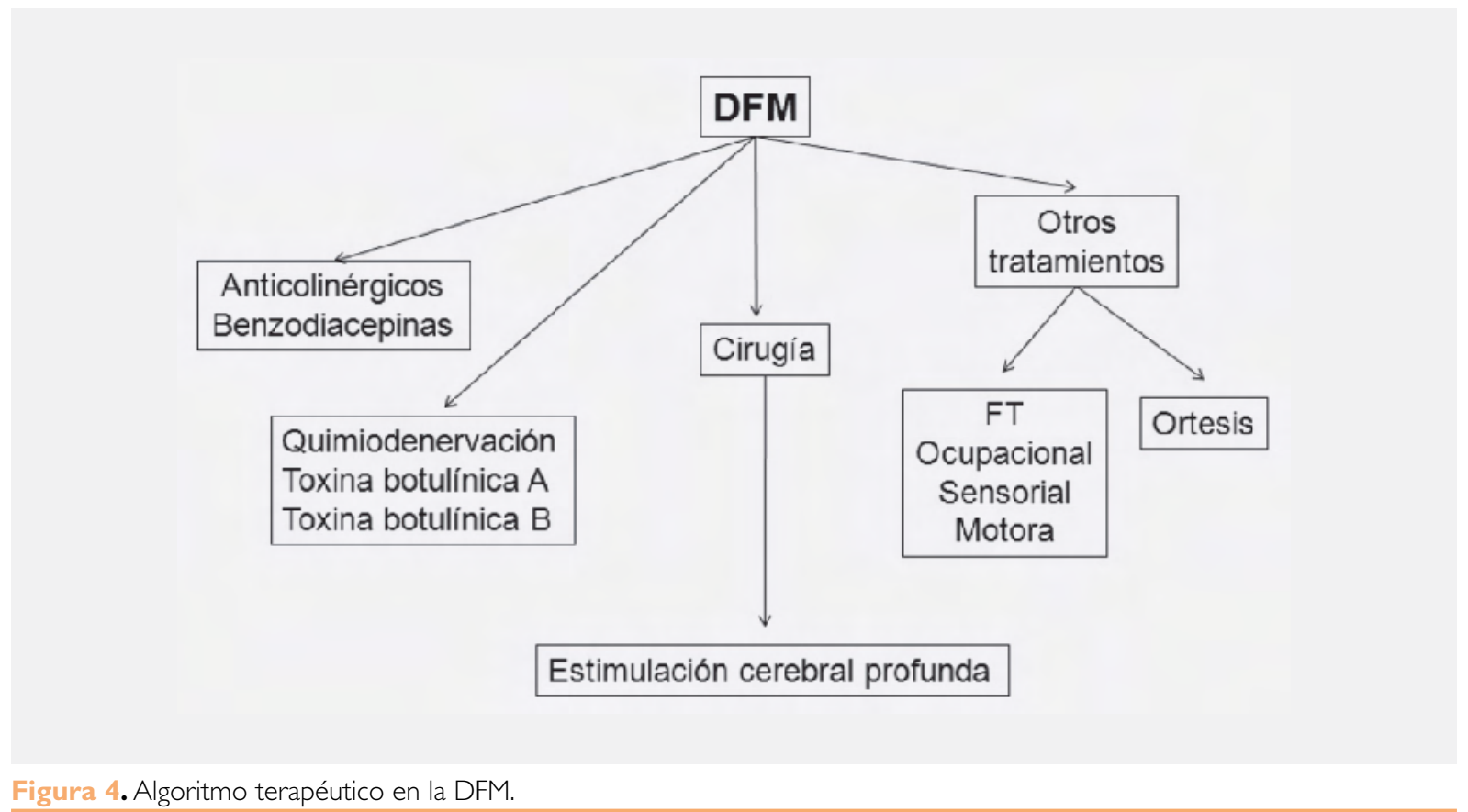




\section{BIBLIOGRAFÍA}

I. Fahn S. Classification of movement disorders. Mov Disord. 20I I; 26: 947-57.

2. Oppenheimer $\mathrm{H}$. Über eine eigenertige Kampfrankheit des kindlichenund jugenlichen aletrs (dysbasia lordotica prpgressiva, dystonie musculorum deformans). Neurol Zentbl. 1911;30:1090107.

3. Citado por: Berrios GE. Musical hallucinations: A historical and clinical study. $\mathrm{Br} J$ Psiquiatry. 1990; 156: | 88-94.

4. Kopp J. Denkwürdigkeiten in der ärtzlichen praxis. Frankfurt, Germany, 1836.

5. Gowers WR. A Manual of Diseases of the Nervous System; P. Blakiston' Son \& Co.:Philadelphia, PA, USA, I888; pp. I059- 1078.

6. Münte TF, Altenmüller $E$, Jäncke I. The musician's brain as a model of neuroplasticity. Natur Rev Neurosciences. 2002;3:473-8.

7. Citado por: Fahn S, Bressman SB, Marsden CD Classification of dystonia. Adv Neurol. 1998;78:110.

8. Citado por: Goetz CG,Vilensky JA. Early cinematographic studies of generalized dystonia. Mov Disord. 2006; 21:156|-5.

9. Nutt JG, Muenter MD, Aronson A. Epidemiology of focal and generalized dystonia in Rochester Minnesota. Mov Disord. 1988;3: 1 88-94.

10. Defazio G, Abbruzzese G, Livrea P, Berardelli A. Epidemiology of primary dystonia. Lancet Neurol. 2004;3:673-8.

I I. Duarte J, Mendoza A, Garcia MT. Epidemiology of primary dystonia. Rev Neurol. 1999;29:884-6.

12. Jabusch HC, Zschucke D, Schmidt A, Schuele S, Altenmuller E. Focal dystonia in musicians: treatment strategies and long-term outcome in 144 patients. Mov Disord. 2005; 20:1623-6.

13. Artieda J, Garcia de Casasola MC, Pastor MA, Alegre M, Urriza J.The pathophysiological basis of dystonia. Rev Neurol. 200 I;32(6):549-58.

14. Aranguiz R, Chana-Cuevas P, Alburquerque D, Leon M. Distonías focales en los músicos. Neurología. 201 I;26:45-52.

15. Hallett M. Neurophysiology of dystonia:The role of inhibition. Neurobiol Dis 201 1;42:177-84.

16. García-Bravo EB, Méndez JL, Sánchez J, Miranda G, Medina F, Hernández Z. Revisión sobre la etiopa- togenia, diagnóstico y tratamiento actual de la distonía ocupacional. Rehabilitación. 2006;40:30-4.

17. Martí i Vilalta JL. Música \& Neurología. Lunwerg SL. Barcelona 20I0, pp. 70.

18. Classen J. Focal hand dystonia - a disorder of neuroplasticity? Brain. 2003; I 26:257 I-2.

19. Bara-Jiménez W, Shelton P, Hallett M. Spatial discrimination is abnormal in focal hand dystonia. Neurology. 2000;55: I 869-73.

20. Bara-Jiménez W, Shelton P, Sanger TD, Hallett M. Sensory discrimination capabilities in patients with focal hand dystonia. Ann Neurol. 2000;47:377380.

21. Tinazzi M, Marotta A, Fasano A, Bove F, Bentivoglio AR, Sqjuintani G, Pozzer L, Fiorio M. Aristotele's illusion reveals inter-digit functional somatosensory alterations in focal hand dystonia. Brain. 2013; 136:782-9.

22. Németh $\mathrm{AH}$. The genetics of primary dystonias and related disorders. Brain 2002; 125: 695-721.

23. De Carvalho Aguiar PM, Ozelius LJ. Classification and genetics of dystonia. Lancet Neurol. 2002; 1:316-25.

24. Phukan J, Albanese A, Gasser T, Warner T. Primary dystonia and dystonia-plus syndromes: cllnical characteristics, diagnosis, and pathogenesis. Lancet Neurol. 201 1; 10:1074-85.

25. Torres-Russotto D, Perlmutter JS. Focal dystonias of the hand and upper extremity. I Hand Surg. 2008:33A: 1657-8.

26. Lederman RJ. Neuromuscular and musculoskeletal problems in instrumental musicians. Muscle Nerve. 2003;27:549-61.

27. Rosset-Llobet J, Molas S, Cubells D, Narberhaus DB, Homs J. Clinical analysis of musicians' focal hand dystonia. Review of 86 cases. Neurologia. 2005;20:108-15.

28. Lee H-S, Park HY, Yoon JO, Kim JS, Chun M, Aminata WA, Cho W-j; Jeon I-H. Musicians' medecine: musculoskeletal problems in string players. Clin Orthop Surg. 2013;5:155-60.

29. Fahn S, Willians D. Psycogenic dystonia. En: Fahn S, Marsden CD, Calne DB. (Eds.). Advances in Neurology: Dystonia 2. $2^{a}$ ed. Raven Press, New York 1988; 50: 431-45।.

30. Jabusch $\mathrm{HC}$, Vauth $\mathrm{H}$, Altenmuller E. Quantification of focal dystonia in pianists using scale analysis. Mov Disord. 2004; 19:171-80. 
31. Tubiana R.Musician's focal dystonia. Hand Clin .2003; 19:303-8.

32. Tubiana R. 'Dystonia Incidence: classification of severity and results of therapy' In: Winspur I and Wynn Parry CB (Eds), The Musician's Hand: A Clinical Guide. Ist edition, Martin Dunitz: London, 1998.

33. Yazawa S, Ikeda A, Kaji R, Terada K, Nagamine T, Toma K, et al. Abnormal cortical processing of voluntary muscle relaxation in patients with focal hand dystonia studied by movement-related potencials. Brain. 1999; I 22: 1 357-66.

34. Pujol J, Roset-Llobet J, Rosines-Cubells D, Deus J, Narberhaus B, Valls-Sole J, Capdevila A, PascualLeone A. Brain cortical activation during guitarinduced hand dystonia studied by functional MRI. Neuroimage. 2000; I 2: 257-67.

35. Zoons E, Booij J, Ne3derveen AJ, Dijk JM, Tijssen MAJ. Structural, functional and molecular imaging of the brain in primary focal dystonia - A review. Neuroimage. 201 1;56:10 1 I-20.

36. Ben-Shlomo Y, Camfi eld L, Warner T. What are the determinants of quality of life in people with cervical dystonia? J Neurol Neurosurg Psychiatry. 2002;72:608-14.

37. Candia V, Schäfer T, Taub E, Rau H, Attenmüller E, Rockstroh, Elbert T. Arch Phys Med Rehabil. 2002;83:1 342-8.

38. Warrington J. Hand therapy for the musician: instrument focused rehabilitation. Hand Clin. 2003; | 9:287-301.

39. Zeuner KE, Bara-Jiménez W, Noguchi PS, Goldstein SR, Dambrosia JM, Hallett M. 'Sensory training for patients with focal hand dystonia'. Annals of Neurology. 2002;5 1:593-8.

40. Zeuner KE, Bara-Jiménez W, Noguchi PS. Sensory training for patients with focal hand dystonia. Ann Neurol. 2002;5 I:593-98.

4I. Kaji R, Kohara N, Katayama M. Muscle afferent block by intramuscular injection of lidocaine for the treatment of writer's cramp. Muscle Nerve. 1995; | 8:234-35.

42. Yoshida K, Kaji R, KuboriT, Kohara N, lizuka T, Kimura J. Muscle afferent block for the treatment of oromandibular dystonia. Mov Disord. 1998; 13:699705.
43. Tas N, Karatas GK and Sepici V. 'Hand orthoses as a writing aid in writer's cramp. Mov Disord. 200।; | 6:1 | 85-9.

44. Adam OR, Jankovic J.Treatment of dystonia. Parkinsonism and Telated Disorders. 2007; 13:362-8.

45. Simpson DM, Blitzer A, Brashear A, Comella C, Dubinsky R, Hallett M, Jankovic J, Karp B, Ludlow $\mathrm{CL}$, Miyasaki JM. Assessment: Botulinum neurotoxin for the treatment of movement disorders (an evidence-based review): Report of the therapeutics and technology assessment subcommittee of the American Academy of Neurology. Neurology 2008;70:1699-706.

46. Dressler D. Clinical applications of botulinum toxin. Curr Opin Microbiol. 20 I2;5:325-336.

47. Chen S. Clinical uses of botulinum neurotoxins: current indications, limitations and future developments. Toxins. 20 I 2;4:9। 3-39.

48. Karp Bl. Botulinum toxin treatment of occupational and focal hand dystonia. Mov Disord. 2004; 19:116-9.

49. Lungu C, Karp Bl, Alter K, Zolbrod R, Hallett M. Long-term follow-up of botulinum toxintherapy for focal hand dystonia: Outcome at 10 years or more. Mov Disord 2011;26:750-753.

50. Karp Bl. Botulinum toxin physiology in focal hand and cranial dystonia. Toxins. 20 I 2;4: I 404- 14.

5I. Boroojerdi B, Cohen LG, Hallett, M. Effects of botulinum toxin on motor system excitability in patients with writer's cramp. Neurology. 2003;61:1543-50.

52. Schuele S, Jabusch HC, Lederman RJ, Altenmuller E. Botulinum toxin injections in the treatment of musician's dystonia. Neurology. 2005;64:34I-3.

53. Karp BI, Cole RA, Cohen LG, Grill S, Lou JS, Hallett M. Long-term botulinum toxin treatment of focal hand dystonia. Neurology. 1994:44:70-6.

54. Bertrand CM, Molina-Negro P. Selective peripheral denervation in II I cases of spasmodic torticollis: rationale and results. In: Fahn S, Marsden CD, Calne DB, eds. Dystonia: advances in neurology, vol 50. New York: Raven Press, 1988: 637-43.

55. Jankovic J. Treatment of dystonia. Lancet Neurol. 2006:5:864-72. 